\title{
Introduction of Fractal Based Information Processing and Recognition
}

\author{
Shuai Liu $1,2, * \mathbb{C}$, Carlo Cattani ${ }^{3}(\mathbb{D})$ and Yudong Zhang ${ }^{4}(\mathbb{D}$ \\ College of Information Science and Engineering, Hunan Normal University, Changsha 410081, China \\ College of Computer Sciences, Inner Mongolia University, Hohhot 010012, China \\ Engineering School (DEIM), Tuscia University, 01100 Viterbo, Italy; cattani@unitus.it \\ 4 School of Computer Science, University of Leicester, Leicester LE1 7RH, UK; yudongzhang@ieee.org \\ * Correspondence: cs.liu.shuai@gmail.com
}

Received: 26 February 2019; Accepted: 1 March 2019; Published: 28 March 2019

Fractal characteristic, one typical nonlinear characteristic, is applied as a key characteristic in complex information processing and used in many research domains [1]. The fractal based characteristics mainly include multifractal dimension, fractional dimensions and so on [2]. Today, fractal based information processing has been successfully applied into the analysis of complex nature or engineering phenomenon, especially the empirical description of information with complex structure [3].

For example, much thermodynamics information can be analyzed by fractal based methods [4]. Moreover, biological information of many species can be discovered by fractal analysis [5]. In addition, fractal based information analysis and processing can be well used in information recognition [6]. In fact, fractal compression can compress information with a very high compressing ratio, which is higher than the classical compression method several times and even more [7]. Fractal based classification and recognition and recognition method are also used in Information Science and Artificial Intelligence [8,9].

Since fractal based methods have started to occupy the central place in information processing and recognition domains today, this theme issue is proposed to provide an opportunity for researchers to publish their gifted theoretical and technological studies of the advanced method in hybrid information processing, and their novel engineering applications within this domain. In this theme issue, nine in 27 submissions are accepted with an accepted ratio of 33.3\%.

The first article, "Extraction of Coal and Gangue Geometric Features with Multifractal Detrending Fluctuation Analysis", authored by Y. Q. Chen from the Mechatronics, Embedded Systems and Automation Lab, University of California, USA, proposed an extraction of the geometric features of coal and gangue by using multifractal detrended fluctuation analysis (MFDFA) method [10]. This method separated the coal and gangue by characteristics of their outline differences, which included multifractal characteristics and modified local singular spectrum widths of outline curve series of coal and gangue. With a combination of traditional grayscale and texture features, the proposed method had large recognition rate than traditional methods.

The second article, "An Automated Segmentation Method for Lung Parenchyma Image Sequences based on Fractal Geometry and Convex Hull Algorithm", authored by Yudong Zhang from the Department of Informatics, University of Leicester, United Kingdom, constructed an automated framework to segment the lung parenchyma images and detect the depression boundary of juxtapleural nodules by combining threshold iteration and fractal geometry [11]. This method finished complete and efficiency segmentation of the juxtapleural nodules with an improved convex hull repair, which was used to complete the accurate segmentation of the lung parenchyma. Evaluation results confirmed that the proposed method can segment juxtapleural lung parenchymal images accurately and efficiently. 
In order to improve rating prediction of tag-aware recommender systems (TRS), the third article titled "TRSDL: Tag-Aware Recommender System Based on Deep Learning-Intelligent Computing Systems", authored by Hai-Tao Zheng from the Tsinghua-Southampton Web Science Laboratory, Tsinghua University, China, investigated a hybrid deep learning model TRSDL (tag-aware recommender system based on deep learning) [12]. They constructed item and user profiles with represented user-defined tags and trained them with a recurrent neural network to extract latent features of both items and users. With deep learning, the proposed method also found advanced implicit features. Experimental results showed that the proposed method significantly outperformed most recent baselines.

The fourth article titled "Synchronization of Chemical Synaptic Coupling of the Chay Neuron System under Time Delay", authored by Jose Tenreiro Machado from the Institute of Engineering, Polytechnic of Porto, Portugal, studies the chemical synaptic coupling of Chay neurons and the effect of adding time delay on their synchronization behavior [13]. They found that coupling strength stimuli affected the discharge activity and the synchronization behavior. In the meantime, the Chay neurons had chaotic discharge behavior in a nonsynchronous state. The system state was changed from asynchronous to synchronous by adding a certain coupling strength or from synchronous to asynchronous by altering a time lag.

To disclose the fractal transformation mechanism of the size distribution in rare earth tailing particles, the fifth article titled "Fractal Behavior of Particle Size Distribution in the Rare Earth Tailings Crushing Process under High Stress Condition", authored by Armando Ciancio from the Department of Biomedical and Dental Sciences and Morphofunctional Imaging, University of Messina, Italy, utilized the side confined uniaxial compression test to study the evolution rule of the particle size distribution of the rare earth tailing and the particle breakage features under the high-stress condition. [14]. With the fractal behavior of the particle size distribution in the crushing process based on fractal model and particle size distribution, their model showed the importance of features under different conditions.

The sixth article titled "Signal Pattern Recognition Based on Fractal Features and Machine Learning", authored by Chang-Ting Shi from the College of Computer Science and Technology, Harbin Engineering University, China, conducted a systematic research study by using the fractal dimension as the feature of modulation signals [15]. They extracted Box, Katz, Higuchi, Petrosian, and Sevcik fractal dimension from eight different modulation signals for signal pattern recognition. Meanwhile, the anti-noise function, box-diagram, and running time are used to evaluate the noise robustness. Experimental results showed the proposed method had better recognition performance, which could reach $96 \%$ in $10 \mathrm{~dB}$ ny using these features into a machine learning method.

The seventh article titled "GARLM: Greedy Autocorrelation Retrieval Levenberg-Marquardt Algorithm for Improving Sparse Phase Retrieval", authored by Guan Gui from College of Telecommunication and Information Engineering, Nanjing University of Posts and Telecommunications, China, proposed an improved phase retrieval algorithm, greedy autocorrelation retrieval Levenberg-Marquardt (GARLM) algorithm, to efficiently solve the non-convex optimization problem with large residual [16]. The GARLM algorithm was a local search iterative algorithm to recover the sparse signal from its Fourier transform magnitude, and the minimizing problem was solved by improved Levenberg-Marquardt (ILM) method.

To study the relationship between soil volume fractal dimension (D) and particle-size classification (PSC) system, the eighth article titled "Different Soil Particle-Size Classification Systems for Calculating Volume Fractal Dimension-A Case Study of Pinus sylvestris var. Mongolica in Mu Us Sandy Land, China", authored by Chengzhong Ma from the Department of Architecture and Landscape Engineering, Dezhou Vocational and Technical College, China, selected topsoil across nine forest density gradients of Pinus sylvestris var. mongolica plantations (MPPs) in the Mu Us sandy land, China [17]. Experiments showed that refining scales of clay and sand fractions could increase D, while the refining scale of silt fraction could decrease D. 
The ninth article titled "Hybrid Models Combining Technical and Fractal Analysis with ANN for Short-Term Prediction of Close Values on the Warsaw Stock Exchange", authored by Michał Paluch from the Institute of Applied Computer Science, Lodz University of Technology, Poland, presents new fractal based methods and models for forecasting stock prices and computing hybrid models [18]. Fractal feature and selected stock market indices such as moving averages and oscillators were extracted as inputs in an artificial neural network (ANN) to predict one day ahead of the closing prices of the assets. Experimental results showed that the hybrid fractal based model outperformed traditional methods and more robust.

Acknowledgments: The guest editors are thankful to Takayoshi Kobayashi, Editor in Chief of Applied Sciences, as well as Assistant Editors of Applied Sciences for their supportive guidance during the entire process. The guest editors would like to thank all authors, the many dedicated referees for their valuable contributions, making this special issue a success.

Conflicts of Interest: The authors declare no conflicts of interest.

\section{References}

1. Liu, S.; Cheng, X.; Fu, W.; Zhou, Y.; Li, Q. Numeric characteristics of generalized M-set with its asymptote. Appl. Math. Comput. 2014, 243, 767-774. [CrossRef]

2. Karaca, Y.; Cattani, C.; Moonis, M.; Bayrak, Ş. Stroke Subtype Clustering by Multifractal Bayesian Denoising with Fuzzy Means and-Means Algorithms. Complexity 2018, 2018, 9034647. [CrossRef]

3. Cattani, C.; Sulaiman, T.A.; Baskonus, H.M.; Bulut, H. On the soliton solutions to the Nizhnik-Novikov-Veselov and the Drinfel'd-Sokolov systems. Opt. Quantum Electron. 2018, 50, 138. [CrossRef]

4. Zhang, Y.; Ranjan Nayak, D.; Yang, M.; Yuan, T.F.; Liu, B.; Lu, H.; Wang, S. Detection of unilateral hearing loss by Stationary Wavelet Entropy. CNS Neurol. Disord.-Drug Targets 2017, 16, 122-128. [CrossRef] [PubMed]

5. Lu, M.; Liu, S.; Sangaiah, A.K.; Zhou, Y.; Pan, Z.; Zuo, Y. Nucleosome positioning with fractal entropy increment of diversity in telemedicine. IEEE Access 2018, 6, 33451-33459. [CrossRef]

6. Shi, B.; Zhang, Y.; Yuan, C.; Wang, S.; Li, P. Entropy analysis of short-term heartbeat interval time series during regular walking. Entropy 2017, 19, 568. [CrossRef]

7. Shuai, L.; Zheng, P.; Xiaochun, C. A Novel Fast Fractal Image Compression Method based on Distance Clustering in High Dimensional Sphere Surface. Fractals 2017, 25, 1740004.

8. Liu, G.; Liu, S.; Muhammad, K.; Sangaiah, A.K.; Doctor, F. Object Tracking in Vary Lighting Conditions for Fog based Intelligent Surveillance of Public Spaces. IEEE Access 2018, 6, 29283-29296. [CrossRef]

9. Liu, S.; Bai, W.; Liu, G.; Li, W.; Srivastava, H.M. Parallel Fractal Compression Method for Big Video Data. Complexity 2018, 2018, 2016976. [CrossRef]

10. Liu, K.; Zhang, X.; Chen, Y. Extraction of Coal and Gangue Geometric Features with Multifractal Detrending Fluctuation Analysis. Appl. Sci. 2018, 8, 463. [CrossRef]

11. Xiao, X.; Zhao, J.; Qiang, Y.; Wang, H.; Xiao, Y.; Zhang, X.; Zhang, Y. An Automated Segmentation Method for Lung Parenchyma Image Sequences Based on Fractal Geometry and Convex Hull Algorithm. Appl. Sci. 2018, 8, 832. [CrossRef]

12. Liang, N.; Zheng, H.-T.; Chen, J.-Y.; Sangaiah, A.K.; Zhao, C.-Z. TRSDL: Tag-Aware Recommender System Based on Deep Learning-Intelligent Computing Systems. Appl. Sci. 2018, 8, 799. [CrossRef]

13. Wu, K.; Wang, D.; Yu, C.; Machado, J.T. Synchronization of Chemical Synaptic Coupling of the Chay Neuron System under Time Delay. Appl. Sci. 2018, 8, 927. [CrossRef]

14. Zhong, W.; Yue, F.; Ciancio, A. Fractal Behavior of Particle Size Distribution in the Rare Earth Tailings Crushing Process under High Stress Condition. Appl. Sci. 2018, 8, 1058. [CrossRef]

15. Shi, C.-T. Signal Pattern Recognition Based on Fractal Features and Machine Learning. Appl. Sci. 2018, 8, 1327. [CrossRef]

16. Xiao, Z.; Zhang, Y.; Zhang, K.; Zhao, D.; Gui, G. GARLM: Greedy Autocorrelation Retrieval Levenberg-Marquardt Algorithm for Improving Sparse Phase Retrieval. Appl. Sci. 2018, 8, 1797. [CrossRef] 
17. Deng, J.; Ma, C.; Yu, H. Different Soil Particle-Size Classification Systems for Calculating Volume Fractal Dimension-A Case Study of Pinus sylvestris var. Mongolica in Mu Us Sandy Land, China. Appl. Sci. 2018, 8, 1872. [CrossRef]

18. Paluch, M.; Jackowska-Strumiłło, L. Hybrid Models Combining Technical and Fractal Analysis with ANN for Short-Term Prediction of Close Values on the Warsaw Stock Exchange. Appl. Sci. 2018, 8, 2473. [CrossRef] 